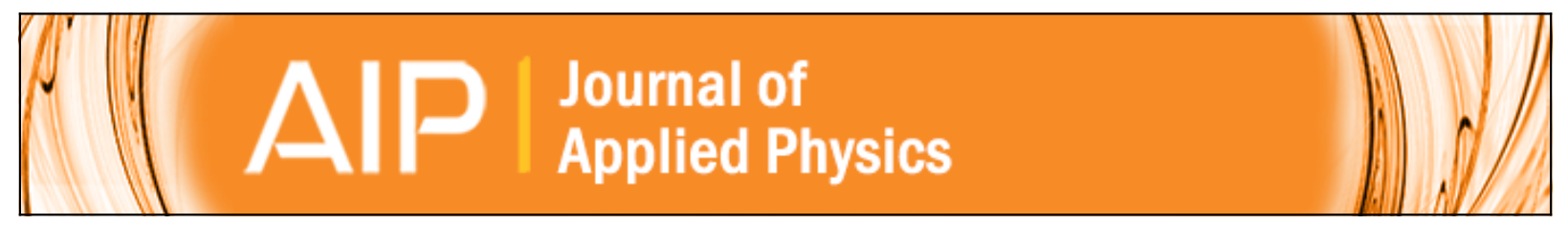

\title{
Anomalous resonance of strontium titanate
}

R. Neville, B. Hoeneisen, and C. A. Mead

Citation: Journal of Applied Physics 43, 3903 (1972); doi: 10.1063/1.1660845

View online: http://dx.doi.org/10.1063/1.1660845

View Table of Contents: http://scitation.aip.org/content/aip/journal/jap/43/10?ver=pdfcov

Published by the AIP Publishing

\section{Articles you may be interested in}

Strain engineered barium strontium titanate for tunable thin film resonators

Appl. Phys. Lett. 104, 202902 (2014); 10.1063/1.4879281

Anomalous capacitance of Schottky barriers on strontium titanate

J. Appl. Phys. 46, 350 (1975); 10.1063/1.321342

Permittivity of Strontium Titanate

J. Appl. Phys. 43, 2124 (1972); 10.1063/1.1661463

Raman Spectrum of Strontium Titanate

J. Chem. Phys. 48, 2240 (1968); 10.1063/1.1669418

Nuclear Magnetic Resonance Study of the Phase Transition in Strontium Titanate

J. Chem. Phys. 38, 726 (1963); 10.1063/1.1733729

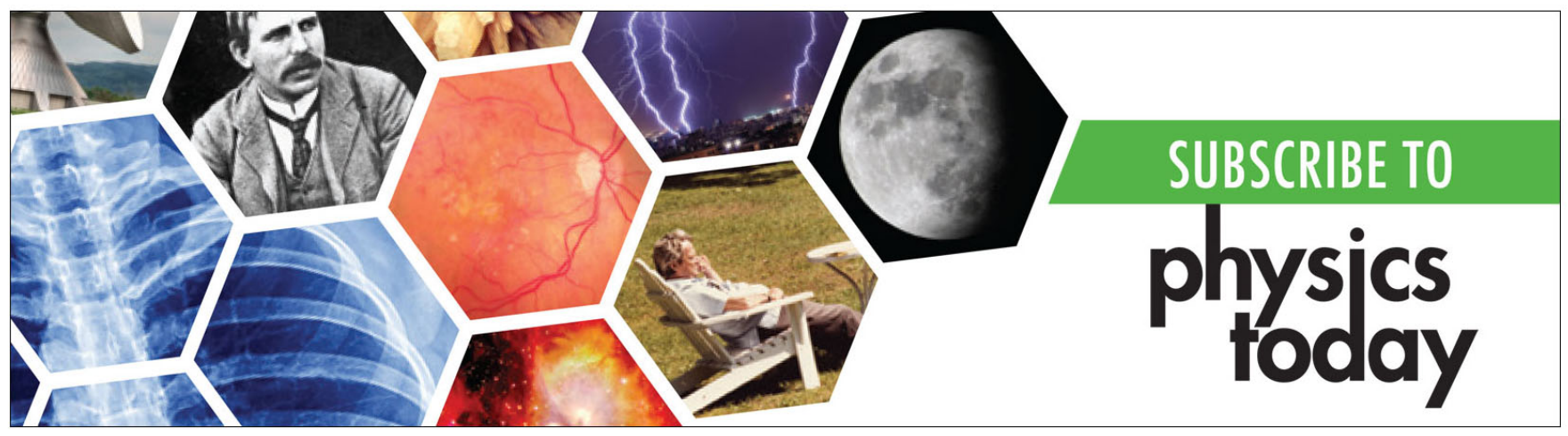




\title{
Anomalous resonance of strontium titanate
}

\author{
R. Neville \\ University of California, Santa Barbara, California 93106 \\ B. Hoeneisen and C. A. Mead \\ California Institute of Technology, Pasadena, California 91109 \\ (Received 14 February 1972; in final form 17 May 1972)
}

An anomalous resonance exhibited by perovskite strontium titanate crystals are obtained by cooling the samples with an applied electric field. A plausible mechanism involves a mechanical oscillation with a frequency determined by the domain boundary spacing, which is coupled to the electric field through the piezoelectric strain constant.

\section{EXPERIMENTAL EVIDENCE}

Wafers of single-crystal perovskite strontium titanate $\left(\mathrm{SrTiO}_{3}\right)$ were prepared as described in Ref. 1. Capaci tors formed by evaporating gold uniformly on one side of the wafers and circular gold contacts on the other side were used to measure the permittivity in the $[100]$, [110], and [111] directions. The wafer thickness was always much smaller than the contact diameter so that fringe fields could be neglected. The apparent relative permittivity is defined by

$$
\epsilon_{r}=U / j \omega \epsilon_{0} A V
$$

where $l$ is the wafer thickness, $A$ is the contact area, $I$ and $V$ are the current and voltage phasors, $\omega$ is the angular frequency, and $\epsilon_{0}$ is the permittivity of free space. The apparent permittivity below $65^{\circ} \mathrm{K}$ is found to be dependent on how the samples are cooled. If the samples are cooled with no applied electric field, the apparent permittivity below $65^{\circ} \mathrm{K}$ is frequency dependent, exhibiting a "resonance" between 1 and $10 \mathrm{MHz}$ as shown in Fig. 1. In the present study we shall be concerned with this anomalous resonance. If the samples are cooled with an applied electric field in excess of $2 \times 10^{4}$ $\mathrm{V} / \mathrm{cm}$, the permittivity is found to be independent of frequency in the measured range from $1 \mathrm{kHz}$ to $50 \mathrm{MHz}$, and equal (within $5 \%$ ) to the low-frequency $\left(<3 \times 10^{5} \mathrm{~Hz}\right)$ permittivity indicated in Fig. 1.

The perovskite strontium titanate lattice is cubic above $110^{\circ} \mathrm{K}$, tetragonal from 110 to $65^{\circ} \mathrm{K}$, and orthorombic below $65^{\circ} \mathrm{K} .{ }^{2}$ The frequency-independent permittivity is also obtained if the electric field (in excess of $2 \times 10^{4}$ $\mathrm{V} / \mathrm{cm}$ ) is applied to the crystal only while cooling through the 110 and $65^{\circ} \mathrm{K}$ phase-transition temperatures (i.e., from $\sim 113$ to $107^{\circ} \mathrm{K}$ and from $\sim 68$ to $63^{\circ} \mathrm{K}$ ). Application of the electric field at other temperatures has no effect on the low-temperature permittivity. If the electric field is applied only while cooling through the $65^{\circ} \mathrm{K}$ phase transition, the complete anomalous resonance is observed. If the electric field is applied only past the $110^{\circ} \mathrm{K}$ phase transition, about $10 \%$ of the anomalous resonance is observed. A single sample can exhibit successively the frequency-dependent and frequency-independent behavior, depending on how the crystal is cooled.

We interpret the foregoing observations as follows: When the cubic crystal is cooled past $110^{\circ} \mathrm{K}$ with an applied electric field, one of the unit cell axes elongates slightly to form a single-domain tetragonal crystal. While cooling the single-domain crystal past $65^{\circ} \mathrm{K}$ with an applied electric field, the remaining two unit cell axes differentiate to form a single-domain orthorombic crystal. If the sample is cooled past $110^{\circ} \mathrm{K}$ with no applied electric field, tetragonal domains form with different $c$-axis orientation. ${ }^{2}$ While cooling the crystal past $65^{\circ} \mathrm{K}$ with no applied electric field, subdomains form with different $a$-axis orientation. We shall refer

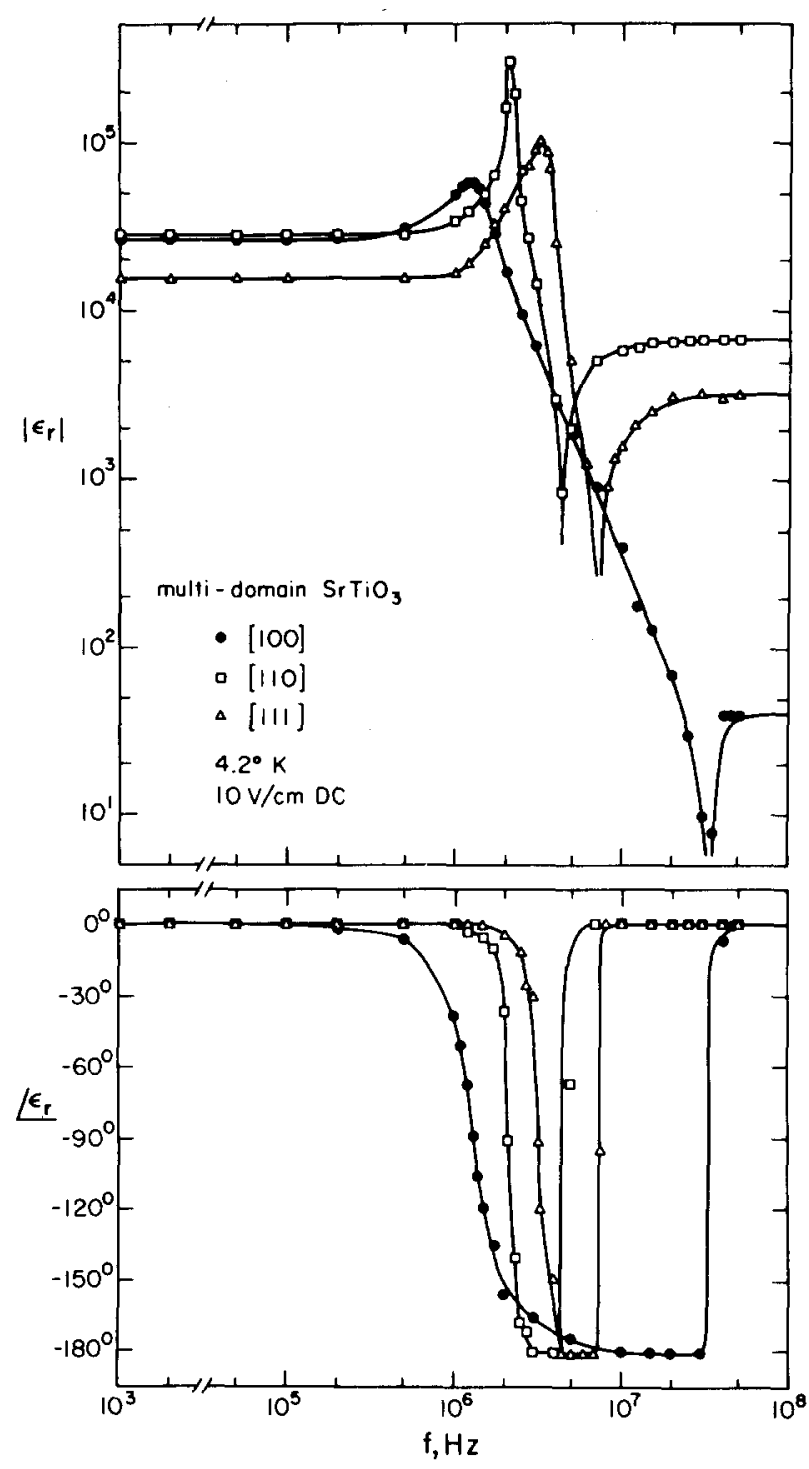

FIG. 1. Magnitude and angle of the appa rent relative permittivity of strontium titanate in the $[100],[110]$, and [111] directions, as a function of frequency. These measurements were made at $4.2^{\circ} \mathrm{K}$ and with an applied dc electric field of $10 \mathrm{~V} / \mathrm{cm}$. These crystals were cooled with no applied electric field. The sample orientation has a one-sigma error of $\pm 1^{\circ}$ and the permittivity has a one-sigma error of $\pm 3 \%$. 


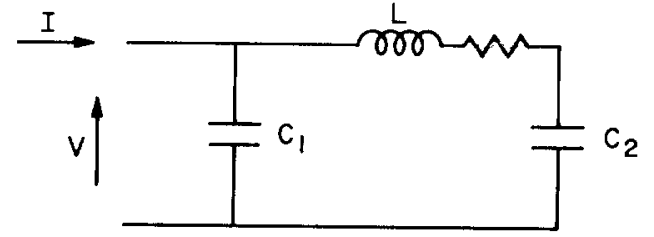

FIG. 2. Equivalent circuit of capacitors with multidomain strontium titanate dielectric below $65^{\circ} \mathrm{K}$.

to the crystals cooled with (without) an applied electric field as single-domained (multidomained).

The curves presented in Fig. 1 were found, within the experimental error, to be independent of the contact diameter, wafer thickness, and wafer area in the measured ranges from 0.1 to $1.2 \mathrm{~cm}, 0.1$ to $1 \mathrm{~mm}$, and 1 to $10 \mathrm{~cm}^{2}$, respectively. In particular, the frequency of the anomalous resonance is not determined by the dimensions of the wafer or the contact. The apparent permittivity was also found to be independent of the amplitude of the ac electric field applied to the crystal, in the measured range from 0.3 to $15 \mathrm{~V} / \mathrm{cm}$ peak. ${ }^{3}$ The phenomenon that causes the anomalous resonance is therefore linear, at least within the measurement range considered above. The curves of Fig. 1 correspond, within experimental error, to the equivalent circuit presented in Fig. 2. The equivalent circuits of three typical samples are summarized in Table I. In the measured directions ([100], [110], and [111]), temperature range (from 4.2 to $300^{\circ} \mathrm{K}$ ), and dc electric field range (from 0 to $\pm 23000 \mathrm{~V} / \mathrm{cm}$ ), the multidomain samples have, within $\pm 5 \%$, the same low-frequency $\left(<3 \times 10^{5} \mathrm{~Hz}\right)$ permittivity as the single-domain crystals. ${ }^{1}$

Changing the experimental setup repeatedly and replacing the samples by various capacitors, we have convinced ourselves that the observed resonance is due to the strontium titanate crystals and not to the experimental setup. Furthermore, the strong dependence of the curves shown in Fig. 1 with crystal orientation together with the good reproductibility of these curves obtained with different samples indicate that the crystals indeed determine the anomalous resonance.

\section{DISCUSSION}

It is difficult to understand what physical phenomena causes the anomalous resonance. In the present discussion we rule out various alternatives and suggest a plausible mechanism.

TABLE I. Contact area $A$ and wafer thickness $l$ of typical multidomain samples oriented in the [100], [110], and [111] directions. The components $C_{1}, C_{2}$, and $L$, and quality factor $Q$ of the equivalent circuit of each sample are specified at $4.2^{\circ} \mathrm{K}$ and a dc electric field of $10 \mathrm{~V} / \mathrm{cm}$.

\begin{tabular}{ccccccc}
\hline \hline & $\begin{array}{c}A \\
\mathrm{~mm}^{2}\end{array}$ & $\begin{array}{c}l \\
\mathrm{~mm}\end{array}$ & $\begin{array}{c}C_{1} \\
\mathrm{pF}\end{array}$ & $\begin{array}{c}C_{2} \\
\mathrm{pF}\end{array}$ & $\begin{array}{c}L \\
\mu \mathrm{H}\end{array}$ & $\begin{array}{c}Q \\
\ldots\end{array}$ \\
\hline$[100]$ & 4.28 & 0.125 & 12.2 & 8010 & 1.87 & $\sim 2.5$ \\
{$[110]$} & 4.28 & 0.125 & 2080 & 6470 & 0.89 & $\sim 10$ \\
{$[111]$} & 4.28 & 0.125 & 970 & 3730 & 0.663 & $\sim 4$ \\
& & & & & & \\
\hline \hline
\end{tabular}

The low-frequency permittivity of strontium titanate calculated using the Lyddane-Sachs-Teller relation, the measured optic-phonon frequencies, and the measured optical permittivity is in quantitative agreement ${ }^{1}$ with the measurements on the single-domain crystals and on the multidomain crystals below $2 \times 10^{5} \mathrm{~Hz}$. The anomalous resonance cannot be attributed to a mechanical oscillation of the crystal as a whole since the resonant frequency is independent of the sample dimensions. The anomalous resonance is not due to an optical phonon since the lowest optical phonon has a natural frequency of $3.2 \times 10^{11} \mathrm{~Hz}$ at $4.2^{\circ} \mathrm{K} .{ }^{1,4}$ Since the anomalous resonance is related to the presence of domains, it is probably due either to domain boundary motion or to a mechanical oscillation with a frequency determined by the domain boundary spacing. If the resonance were due to domain boundary motion, motion of the domain boundaries would be expected below the resonance frequency and not above it. The permittivity of single-domain and multidomain samples would therefore be equal above the resonant frequency, contrary to experiment. We have therefore assumed that the resonance is due to a mechanical oscillation with a frequency determined by the domain boundary spacing. The resonant frequency $f_{0}$ is then of the order of $\left(s \rho L^{2}\right)^{-1 / 2}$, where $\rho$ is the density, $L$ is the domain boundary spacing, and $s$ is the "effective" elastic compliance constant of the particular mode of oscillation. Using a typical domain boundary spacing of $10 \mu \mathrm{m}^{2,5}$ and a resonant frequency of $2 \mathrm{MHz}$ (see Fig. 1) one obtains $1 / \mathrm{s} \approx 2 \times 10^{6} \mathrm{~N} / \mathrm{m}^{2}$ at $4.2^{\circ} \mathrm{K}$, which is smaller than the elastic stiffness constants $c_{11}, c_{12}$, or $c_{44}$ (measured above $110^{\circ} \mathrm{K}$ ) by a factor of $\approx 10^{5}$. A cubic crystal subject to a tensile stress in the $[100] \mathrm{di}-$ rection has a Young's modulus $1 / s=\left(c_{11}^{2}+c_{11} c_{12}-2 c_{12}^{2}\right) /$ $\left(c_{11}+c_{12}\right)$ and a Poisson ratio $c_{12} /\left(c_{11}+c_{12}\right)$. Notice that if $c_{11}=c_{12}, 1 / s=0$, the lattice becomes unstable and the crystal is free to undergo the cubic to tetragonal transition. In this transition the volume of the unit cell is conserved to first order in the strain. Measurements indicate that $c_{11}$ approaches $c_{12}$ as the temperature approaches $110{ }^{\circ} \mathrm{K}$ from above. ${ }^{6}$ Since from 110 to $4.2^{\circ} \mathrm{K}$ the crystal undergoes a second (and perhaps a thir $\mathrm{d}^{2}$ ) phase transition, it is likely that the crystal lattice is marginally stable in this temperature range. The low value predicted for $1 / s$ at $4.2^{\circ} \mathrm{K}$ is therefore possible.

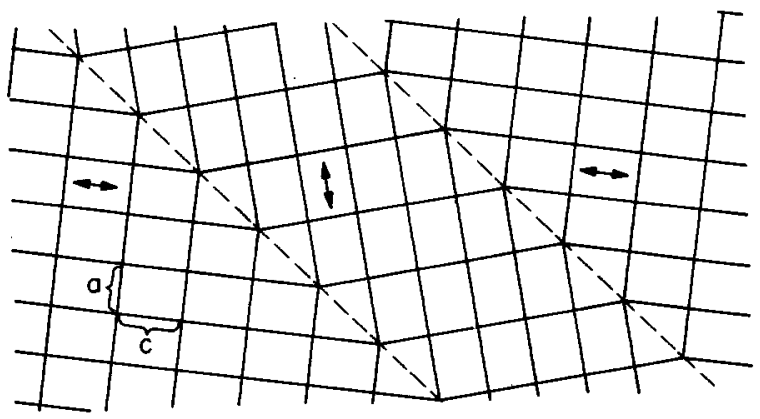

FIG. 3. Lattice of multidomain strontium titanate (schematic). Two domain boundaries are shown. The arrows indicate the [100] direction. 


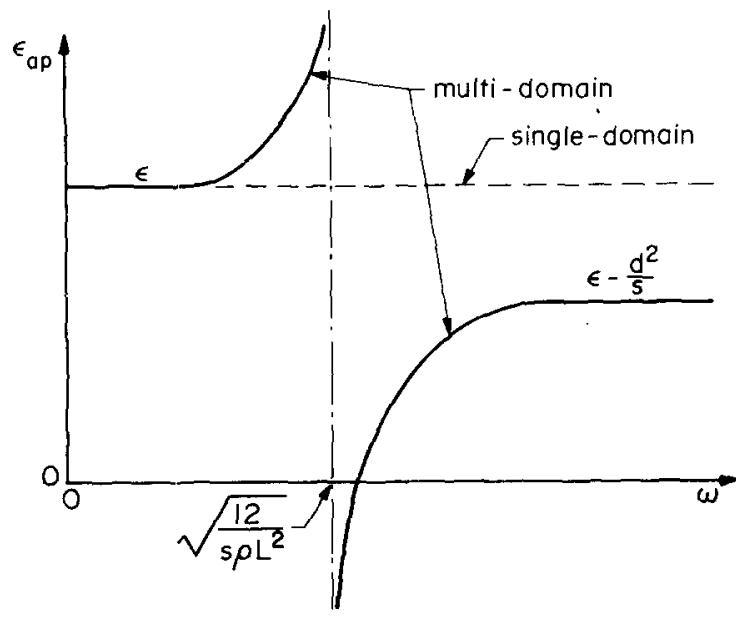

FIG. 4. Apparent permittivity of multidomain strontium titanate as a function of angular frequency, derived from Eq. (7).

The lattice of a multidomain crystal is presented schematically in Fig. 3. Notice that in the cubic to tetragonal transition two adjacent domains must "rotate" with respect to each other by $2 \alpha \approx(c-a) / c$ in order that the two domains coincide at the domain boundary. ${ }^{7}$ The anomalous resonance is attributed to the "soft" oscillation in which the $c / a$ ratio, and therefore $\alpha$, vary with time. The kinetic energy per unit volume of such an oscillation is

$$
T=\frac{1}{2} \rho\left(L^{2} / 12\right) \dot{\alpha}^{2}=\frac{1}{2} \rho\left(L^{2} / 12\right) \dot{e}^{2},
$$

where $e$ is the elastic strain. This oscillation can couple to the electric field if the crystal is ferroelectric and/ or piezoelectric. If the crystal were ferroelectric, the low-frequency permittivity of multidomain crystals would be greater than that of single-domain crystals. This difference in permittivity is because the lattice in the multidomain crystal can "rotate" with respect to the electric field, whereas it cannot in the single-domain sample. Since the low-frequency permittivities are found to be equal, we conclude that the anomalous resonance is not due to ferroelectricity. Furthermore we have found no evidence of ferroelectricity. ${ }^{1}$ We therefore assume that the multidomain crystals are piezoelectric as indicated by Rupprecht and Winter. ${ }^{8}$ In schematic one-dimensional notation a piezoelectric crystal is described by the following equations ${ }^{7}$ :

$$
P=\sigma d+E \chi, \quad e=\sigma s+E d,
$$

where $P$ is the polarization density, $\mathrm{C} / \mathrm{m}^{2} ; \sigma$ is the stress, $\mathrm{N} / \mathrm{m}^{2} ; d$ is the piezoelectric strain constant, $\mathrm{m} / \mathrm{V} ; E$ is the macroscopic electric field, $\mathrm{V} / \mathrm{m} ; \chi$ is the dielectric susceptibility, $\mathrm{F} / \mathrm{m}$; $e$ is the elastic strain, -; $s$ is the elastic compliance constant, $\mathrm{m}^{2} / \mathrm{N}$.

Neglecting losses, the increment in kinetic energy per unit volume $\delta T$ is equal to the work per unit volume done by the stress $-\sigma \delta e$. This equality determines the differential equation

$$
\rho\left(L^{2} / 12\right) \ddot{e}+(1 / s) e=E d / s,
$$

$$
e=\frac{E d}{1-\omega^{2} \rho\left(L^{2} / 12\right)_{S}} .
$$

The current density is given by

$$
J=\frac{\partial}{\partial t}\left(P+\epsilon_{0} E\right),
$$

which (in the frequency domain) is

$$
J=j \omega \epsilon E-j \omega \frac{d^{2}}{s} E+\frac{j \omega\left(d^{2} / s\right) E}{1-\omega^{2} \rho\left(L^{2} / 12\right)_{S}},
$$

where $\epsilon \equiv \epsilon_{0}+\chi$ is the permittivity of the single-domain crystal. Notice that Eq. (7) can be represented by the equivalent circuit of Fig. 2. The apparent permittivity derived from Eq. (7) is plotted in Fig. 4. We should note that in a complete analysis the $s$ and $d$ in Eq. (7) are actually functions of the tensor components $s_{i j k t}$ and $d_{i j k}$, respectively, ${ }^{9}$ and that the $s$ in $12 / s \rho L^{2}$ and in $d^{2} / s$ (see Fig. 4) are different in general.

\section{CONCLUSION}

An anomalous resonance which perovskite strontium titanate crystals exhibit below $65^{\circ} \mathrm{K}$ is described. The resonance is attributed to the presence of domains. We have shown that single-domain crystals can be formed by cooling the samples past the 110 and $65^{\circ} \mathrm{K}$ phase transition temperatures with an applied electric field in excess of $2 \times 10^{4} \mathrm{~V} / \mathrm{cm}$. The sample becomes single domained possibly because the multidomained tetragonal crystal is piezoelectric. Other workers have formed single-domain crystals by cooling the samples past the transition temperatures with an applied uniaxial stress. ${ }^{5}$

The anomalous resonance is difficult to understand and we tentatively offer the following explanation: The elastic stiffness constants $c_{11}$ and $c_{12}$ approach each other as the crystal is cooled to $110^{\circ} \mathrm{K}$. When $c_{11}=c_{12}$ the lattice becomes unstable and the cubic to tetragonal transition occurs. If $c_{11} \approx c_{12}$, the Young's modulus in the [100] direction is small and little energy is required to change the $c / a$ ratio of the unit cell (keeping the volume constant to first order in the strain). An oscillation in which the $c / a$ ratio varies with time can account for the observed anomalous resonance if the multidomain crystal is piezoelectric. The frequency of the resonance would be determined by the domain boundary spacing. Additional experimental evidence is required to have a more reliable and detailed understanding of the phenomenon.

'R. Neville, B. Hoeneisen, and C. A. Mead, J. Appl. Phys. 43, 2124 (1972) ${ }^{2}$ F. W. Lytle, J. Appl. Phys. 35, 2212 (1964)

${ }^{3}$ Except that the peak of the resonance is $10 \%$ lower with an applied ac electric field of $15 \mathrm{~V} / \mathrm{cm}$ than with $0.3 \mathrm{~V} / \mathrm{cm}$ peak.

${ }^{4} \mathrm{~J}$. M. Worlock and P. A. Fleury, Phys. Rev. Lett. 19, 1176 (1967).

${ }^{5}$ T. S. Chang, J. F. Holzrichter, G. F. Imbusch, and A. L. Schawlow, Appl. Phys. Lett. 17, 254 (1970).

${ }^{6}$ R. O. Bell and G. Rupprecht, Phys. Rev. 129, 90 (1963).

'If the interpretation of the domains indicated in Fig. 3 is correct, the Young's

modulus of multidomain samples is much larger than that of single-domain

samples in the [100] direction, when $c_{11} \approx c_{12}$. The elastic constants of strontium titanate below $110^{\circ} \mathrm{K}$ should therefore be determined using single-domain crystals.

${ }^{B}$ G. Rupprecht and W. H. Winter, Phys. Rev. 155, 1019 (1967).

${ }^{9}$ J. F. Nye, Physical Properties of Crystals (Oxford U.P., Oxford, England, 1957). 